\title{
LEJOS: LA DISTOPÍA SOCIALISTA-FEMINISTA DE CARYL CHURCHILL
}

\author{
Paola Sotomayor-Botham \\ Birmingham City University \\ paola.botham@gmail.com
}

\section{RESUMEN / ABSTRACT}

Lejos, una de las dos únicas obras de Caryl Churchill que se han montado en Chile, presenta una visión alarmante del siglo XXI que combina la guerra y el desastre ecológico. En la dramaturgia de esta autora inglesa, sin embargo, la presencia de un futuro aterrador opera como advertencia para estimular el cambio social. Usando elementos de Teoría Crítica Feminista, así como también la distinción literaria entre 'distopía' y 'anti-utopía', este artículo sostiene que -pese a ciertas ambigüedades detectadas por la crítica- el trabajo de Churchill no ha abandonado sus ideales políticos progresistas.

Palabras Clave: Caryl Churchill, Lejos, distopía, feminismo, teatro político.

Far Away, one of only two plays by Caryl Churchill that have been performed in Chile, offers an alarming vision of the $21^{\text {st }}$ century, which combines war and ecological disaster. In the theatre of this English dramatist, however, the presence of a frightening future operates as a warning in order to encourage social change. Using elements of Critical Feminist Theory as well as the literary distinction between 'dystopia' and 'anti-utopia', this article argues that-despite certain ambiguities that have been detected by critics-Churchill's work has not abandoned its progressive political ideals.

KEY WORDS: Caryl Churchill, Far Away, dystopia, feminism, political theatre.

Far Away, de la dramaturga inglesa Caryl Churchill (1938), se estrenó en Londres en noviembre de 2000. Bajo el título Lejos -en traducción de Camila Le-Bert y Alicia del Campo- esta obra llegó a Chile casi una década más 
$\operatorname{tarde}^{1}$, sin haber perdido un ápice de su escalofriante relevancia. Churchill desarrolló el texto después de tres años de silencio teatral, con una visión casi profética. Escrita antes de los ataques de septiembre de 2001 en Estados Unidos y las subsecuentes campañas militares en Afganistán e Irak, y cuando el cambio climático no era todavía un tema político dominante, Lejos presenta un escenario apocalíptico que combina dos de las preocupaciones fundamentales de principios del siglo XXI: conflicto global y desastre ecológico. La acción se desarrolla en tres actos muy breves organizados en estricto orden cronológico, que muestran tres momentos significativos en la vida de Joan, el personaje protagónico. Al tiempo que ella se transforma de niña curiosa en joven conformista y, más tarde, en soldado, el mundo a su alrededor involuciona desde atrocidades en una casa a una guerra generalizada en la que países, profesiones, animales e incluso los elementos se atacan unos a otros sin razón aparente.

La versión estrenada en Santiago por la compañía Teatro del Carmen en junio de 2010 quiso darle a la obra un contexto explícitamente político. De acuerdo a la actriz y dramaturga Camila Le-Bert, cuya traducción conserva los nombres ingleses de los personajes pero los dota de un habla reconocidamente nacional, el espacio de Lejos es el "Chile neoliberal de la post-post-dictadura" y su tiempo, "el momento histórico [...] del bicentenario y del primer Presidente de derecha elegido democráticamente en 52 años". En mi opinión, esta estrategia de adaptación es apropiada, ya que el trabajo de Churchill se caracteriza por su contenido político, aun en piezas como esta que rehúyen una lectura directa. A pesar de que Churchill es considerada por muchos como la más notable dramaturga británica viva, Lejos es solo la segunda de sus obras que se monta en Chile. A través de su análisis, este artículo busca por tanto ofrecer una visión general y actualizada del teatro de Churchill, en la que el recurso distópico de un futuro aterrador se usa no para negar sino para reafirmar ideales de igualdad social y de género ${ }^{2}$.

\footnotetext{
Agradezco a Camila Le-Bert por facilitarme el manuscrito de Lejos, que es el texto usado en este artículo. Las traducciones del resto de las fuentes en inglés son mías.

Este artículo es un extracto, traducido y adaptado, de mi tesis doctoral: Botham, Paola; "Redefining Political Theatre in Post-Cold War Britain (1990-2005): An Analysis of Contemporary British Political Plays"; Coventry University, 2009. Parte del material fue presentado también como ponencia en el simposio internacional Churchill Now: 21 st Century Caryl Churchill; University of Lincoln, 16 abril 2011, bajo el título "Not Far Away: Caryl Churchill's $21^{\text {st }}$-Century Feminism".
} 


\section{DE LO DOMÉSTICO A LO GLOBAL}

La trama de Lejos se desarrolla como una pesadilla a la inversa: Joan despierta para vivir la catástrofe en lugar de escapar de ella, a la vez que el tiempo escénico - que abarca varios años- se mueve desde la "noche" en el primer acto al "día" en el tercero, ambos situados en la casa de campo de Harper, tía de Joan (Churchill, Lejos 3, esc. 1; 19, esc. 3). En la primera secuencia, Harper envía a Joan a la cama después de que esta ha presenciado una realidad brutal de la que no debía enterarse. En la última, Joan emerge una vez más desde el sueño, ahora como participante activa en el horror ya inevitable de la violencia perpetua y la devastación ambiental.

De acuerdo con Amelia Howe Kritzer, la trayectoria estilística de la obra va del realismo al expresionismo y de ahí al absurdo (Political Theatre 72). De hecho, a pesar de la barbarie que se adivina en el primer segmento, éste no abandona el registro naturalista. El personaje de Joan (que en el montaje londinense estuvo a cargo de una niña real en lugar de una actriz adulta) gradualmente revela las escenas perturbadoras que ha visto en la bodega de su tío, mientras que Harper intenta convencerla de que todo es normal. Joan se las arregla para refutar cada una de las explicaciones de Harper, que incluyen un pájaro, una fiesta, el atropello de un perro, hasta que esta se ve obligada a admitir su participación en cierto tráfico de refugiados que nunca se explica a cabalidad. Sin embargo, Harper insiste en encubrir la verdad hasta el último momento y Joan finalmente acepta su versión de los hechos:

JOAN ¿Por qué mi tío les estaba pegando?

HARPER ¿Pegándole a quién?

JOAN Le estaba pegando a un hombre con un palo. Creo que era un palo de metal. Le pegó a uno de los niños.

HARPER Una de las personas en el camión era un traidor. No era realmente uno de ellos, se estaba haciendo, los iba a traicionar, ellos se enteraron y le contaron a tu tío. Después él atacó a su tío, atacó a los demás y tu tío tuvo que pelear con él.

JOAN Por eso había tanta sangre.

HARPER Sí, había que hacerlo para salvar a los demás.

JOAN Le pegó a uno de los niños.

HARPER Ese tendría que haber sido el hijo del traidor. O incluso a veces hay niños malos que hasta traicionan a sus papás ( 9 ; esc. 1$)$. 
Dada la serie de mentiras que Harper ya le ha contado a Joan y su justificación soez para el uso de violencia contra menores, este pasaje es, a todas luces, una falsedad más. No obstante, Harper convence a Joan de que ahora ella es "parte de un gran movimiento para mejorar las cosas" y Joan se convierte en cómplice al aceptar volver a dormir e incluso "ayudar a limpiar en la mañana" (10; esc. 1).

En el segundo acto, han pasado varios años y Joan está iniciando su trabajo en una sombrerería, donde conoce a su colega y futuro esposo, Todd. El naturalismo de la primera parte es inmediatamente truncado con la información de que los dos estudiaron "sombrero" en la universidad (11; esc. 2.1). Breves escenas muestran a Joan y Todd trabajando durante cuatro días seguidos, a medida que los sombreros pasan de "decorados vivamente" (12; esc. 2.2) a "muy grandes y extravagantes" (13, esc. 2.3$)$, para terminar en "enormes y ridiculos" (15; esc. 2.4). En el quinto día de este exceso expresionista, el propósito siniestro de los sombreros se da a conocer. La didascalia dice: "Una procesión de prisioneros andrajosos, machucados y encadenados, cada uno lleva puesto un sombrero camino a su ejecución" (16, esc. 2.5). Churchill sugiere el mayor número de personas posible para este macabro desfile: "cinco son muy pocas y veinte es mejor que diez. ¿Cien?" (2). Tras la conmoción de esta escena (al menos para los espectadores), Joan y Todd están de vuelta en el trabajo, impasibles, concentrados en nuevas creaciones y en su mutuo enamoramiento. Lo único que Joan lamenta es que la mayoría de los sombreros sean quemados "con los cuerpos" (17; esc. 2.6). Todd tiene planes de denunciar prácticas corruptas en la industria del sombrero, pero esta causa suena ahora insignificante en comparación con el uso que se hace del producto y que a nadie inmuta.

Años después, en el último acto, ya es demasiado tarde para cambiar las cosas. En la casa de campo, Harper y Todd hablan de una guerra equívoca en la que no solo países, sino también insectos, animales, distintas profesiones e incluso "niños menores de 5 " forman alianzas inestables (20; esc. 3). Como prueba de su confiabilidad, Todd enumera: "le he disparado a ganado y niños en Etiopía [...] gaseado a tropas mixtas de españoles, programadores informáticos y perros [...] desgarrado a estorninos con mis manos" (22; esc. 3). Joan, que mató "a dos gatos y un niño menor de cinco" en su camino, está descansando después de un largo viaje, con el fin de pasar al menos un día con su marido. Cuando despierta, su turbador relato detalla cómo todo en el medio ambiente se ha convertido en un enemigo o un arma. Los japoneses controlan el tiempo; los bolivianos, la gravedad; hay soldados chilenos al acecho, y no se puede saber de qué lado está el río (24; esc.3). 
Este giro final hacia el mundo del absurdo encontró una respuesta mixta entre los críticos británicos. Una de las reseñas más negativas fue la de Charles Spencer en el Daily Telegraph: "La visión de Churchill [...] emerge más bien tonta que terrorífica. Cuando nos enteramos de que 'los elefantes se han pasado al bando holandés', resulta imposible contener la risa" (1576). Esta hostilidad hacia una ruptura estilística repentina se asemeja a la recepción crítica del controvertido estreno de Blasted (1995), de Sarah Kane ${ }^{3}$. En el diario Guardian, Michael Billington estableció una comparación explícita entre ambos montajes: "el viaje de la realidad de la primera escena al caos cósmico de la última es demasiado rápido para que sea dramáticamente convincente: me recuerda, en todo caso, a Blasted de Sarah Kane, que sé que Churchill admira pero que a mí me parece un prototipo cuestionable" $(1578)^{4}$. Pese a que, a diferencia de Blasted, en Lejos toda la violencia ocurre fuera de escena, ambas obras tienen bastante en común, principalmente en términos de su estructura -que transita súbitamente de lo doméstico a lo global- y de su negativa a ofrecer explicaciones que justifiquen la acción dramática. Otros comentarios de los críticos reflejan esta última característica. Por ejemplo, “¿Es esta una obra sobre el totalitarismo o la ecología y, si es así, qué dice acerca de estos temas?" (Koenig 1576), o bien, "[Esta obra] no da argumentos, simplemente presenta; no se desarrolla, sino que salta de un punto a otro" (Clapp 1576). En términos políticos, sin embargo, la pieza de Churchill ofrece mucho más que la de Kane, especialmente si se la inserta en el contexto del movimiento feminista.

\section{LO PERSONAL Y LO POLÍTICO}

La historiadora Margot Canaday usa la frase "Teoría Crítica Feminista" para describir el trabajo de Nancy Fraser y Seyla Benhabib, cuya posición filosófica "combina los ideales universales y juicios normativos de la modernidad con el contextualismo, la particularidad y el escepticismo de la postmodernidad" (65). Canaday emplea esta denominación para hacer

\footnotetext{
Con el título Devastados, esta obra se montó en Chile en 2002 bajo la dirección de Alfredo Castro.

4 Como muchos otros críticos, Billington cambió de idea acerca de Blasted después de su reposición en 2001, reconociendo los méritos artísticos de Kane (1971-1999).
} 
hincapié en el enfoque ecléctico de Fraser y Benhabib, similar a la filosofía heterodoxa e interdisciplinaria de la Escuela de Frankfurt (también conocida como Teoría Crítica). Ambas pensadoras reconocen una afinidad directa con esta tradición neo-marxista de pensamiento. La etiqueta de Canaday es útil para designar a un tipo de feminismo que ha logrado superar productivamente los impasses teóricos del pasado.

La crítica de la separación entre lo público y privado, encapsulada en el viejo lema 'lo personal es político', ha sido y sigue siendo una preocupación clave para la teoría feminista. De acuerdo con Carol Pateman, este problema "resulta fundamental en casi dos siglos de literatura y lucha política [...] es, en última instancia, de lo que se trata el movimiento" (103). Dentro del movimiento, sin embargo, se pueden encontrar posiciones divergentes respecto de este tema. Para Nancy Fraser, por ejemplo, el proyecto feminista no es "el colapso de las fronteras entre lo público y lo privado", sino la superación "de la jerarquía de género que da a los hombres más poder que a las mujeres para trazar la línea entre lo público y lo privado" ("Politics, Culture and the Public Sphere" 305). Esta metáfora de trazar una línea es significativa, ya que evita dos posturas extremas: una frontera fija (la línea dibujada de antemano) o un límite confuso (sin línea clara). En concordancia, Seyla Benhabib enfatiza la posibilidad de "hacer públicos" temas que pueden no ser inmediatamente visibles como tales; hacerlos "accesibles para el debate, la reflexión, la acción y la transformación político-moral" ("Models of Public Space" 94). En otras palabras, no se trata simplemente de declarar que lo personal es político, sino que lo personal tiene que hacerse político cuando cuestiones que atañen al interés común están en juego. Fraser sostiene que en nuestra era "post-socialista", las demandas culturales (es decir, la lucha por el reconocimiento de identidades) tienden a eclipsar a las demandas sociales y económicas de redistribución. Lo que se necesita entonces es una combinación de "redistribución y reconocimiento" (Justice Interruptus 12), para lo cual el feminismo debe "desarrollar una visión que se oponga a la desigualdad social y al androcentrismo cultural simultáneamente" (177).

El punto de vista de Benhabib, a su vez, proviene de una crítica constructiva a la "ética del discurso", paradigma desarrollado por Jürgen Habermas y Karl-Otto Apel de la Escuela de Frankfurt. En términos generales, esta postura permite una defensa de una teoría moral universalista en el contexto de sociedades pluralistas modernas. Siguiendo el modelo kantiano y en contra del relativismo, la ética del discurso sugiere que es posible evaluar 
normas socialmente aceptadas como buenas o malas. Sin embargo, el criterio para evaluar proposiciones normativas no tiene lugar, como sugiere Kant, dentro de la mente individual, sino que en un proceso real de argumentación intersubjetiva (esto es, en público). Una de las objeciones más influyentes a las teorías morales universalistas ha sido formulada por la psicóloga feminista Carol Gilligan, quien -desde una base empírica- llegó a la conclusión de que el desarrollo moral de las mujeres tendía a seguir un curso diferente al propuesto por la ética del discurso: "el problema moral surge del conflicto de responsabilidades, no de derechos, y requiere para su resolución un modo de pensar contextual y narrativo en lugar de formal y abstracto" (19). Esta concepción puede ser descrita como una "ética del cuidado", sustentada en lo psicológico y en las relaciones humanas, que contrasta con la lógica formal de la justicia (73). Llevada al extremo, no obstante, una ética del cuidado puede desembocar en lo que Mary Dietz llama "pensamiento maternal", que erróneamente utiliza "el amor y la intimidad" como base de la acción política y el discurso (32).

Benhabib emplea la ética del cuidado de Gilligan, pero se las arregla para evitar la trampa del pensamiento maternal. Su solución es una versión revisada de la ética del discurso, que ella llama "universalismo interactivo" y que complementa el punto de vista "generalizado" de las teorías morales universalistas con el "concreto" sugerido por Gilligan. El primero "nos obliga a ver a todos y cada uno de los individuos como seres racionales, con los mismos derechos y deberes que nos atribuimos a nosotros mismos". El segundo, en cambio, "nos obliga a ver a cada ser racional como un individuo con una historia concreta, una identidad y una constitución afectivo-emocional". En el primer caso, las normas son "públicas e institucionales"; en el segundo, son normas "privadas, no institucionales" de "amistad, amor y cuidado" ("The Generalized and the Concrete Other" 81, 87). Finalmente, Benhabib argumenta que los "dilemas del cuidado son también cuestiones morales y pueden ser tratadas desde una perspectiva universalista" ("The Debate Over Women and Moral Theory Revisited" 189).

La dramaturgia de Churchill se puede considerar como realización artística de estas ideas, en cuanto los límites de lo personal/privado y lo político/público se interrogan constantemente, incluyendo el amor maternal y su relación con el resto de la sociedad. En Lejos, cuando Todd defiende a los cocodrilos porque "cuidan a los cocodrilos bebés y los llevan al agua en sus bocas", Harper responde mordazmente: “¿No crees que todo el mundo ayuda a sus propios niños?" (21; esc. 3 ). 


\section{CHURCHILL: ¿SOCIALISTA-FEMINISTA?}

En una carrera que abarca cinco décadas, el deseo de experimentar de Churchill ha producido una dramaturgia en permanente transformación, donde casi nada se puede dar por sentado. No obstante, existe una constante que sustenta su trabajo. Como señala Elaine Aston, "Churchill es una dramaturga cuya trayectoria y perspectiva política han sido constituidas conscientemente desde un compromiso incesante con el feminismo y el socialismo" (Feminist Views 18). Esta aseveración ha sido, sin embargo, cuestionada en innumerables ocasiones. Jane Thomas y Daniel Jernigan, por ejemplo, se basan en la asociación explícita de Churchill con la filosofía de Foucault en la obra Softcops (1978) para interrogar interpretaciones que siguen una agenda socialista-feminista. Thomas alega que tales lecturas "son a menudo incapaces de dar cuenta de ciertas lagunas y contradicciones en los textos [de Churchill,] excepto como omisiones, aberraciones o, en algunos casos, traiciones del paradigma político" (160). Jernigan está de acuerdo: "Yo veo toda esta ambivalencia en cuanto a las simpatías políticas de Churchill como un signo de que ella comprende la manera en que las paradojas de la ideología frenan la potencialidad de cambio". Lo dicho convertiría a Churchill en una escritora postmodernista, que resiste el sistema pero al mismo tiempo está implicada en él (40). Las dudas planteadas por Foucault frente al concepto de verdad son fundamentales en las apreciaciones de Thomas y Jerningan con respecto a Churchill. Ambos mantienen que su dramaturgia responde a la afirmación de Foucault de que "la 'verdad' está vinculada en una relación circular con los sistemas de poder que la producen y la sostienen" (Cit. en Jernigan 34). En palabras de Thomas, las obras de Churchill "desafían la noción misma de verdad, y las relaciones de poder que la construyen en la edad moderna" (162).

La impresión de que Churchill se ha movido gradualmente del 'activismo' a la 'deconstrucción' es bastante generalizada. Sin embargo, esta perspectiva tiende a subestimar la energía política de sus obras recientes. Si bien es evidente que Churchill ha ido dejando atrás una dramaturgia predominantemente brechtiana para dar paso a una variedad de estilos (Aston, Feminist Views 19), sería reduccionista entender esta transición como un abandono de ciertos ideales políticos. El trabajo de Churchill ha sido siempre complejo. Incluso en sus obras tempranas, el impulso activista o de emancipación tiende a ir de la mano con uno deconstructivo, en el sentido de desmantelar soluciones fáciles. Por otra parte, aunque en muchas sus obras de la década de 1990 las 
estrategias utilizadas pueden ser descritas como postmodernas, gran parte de la experimentación de Churchill -incluyendo el absurdismo de Lejos-se pueden ubicar cómodamente dentro del modelo modernista. Al considerar continuidades y discontinuidades en el trabajo de Churchill, se puede argumentar que su teatro no ha renunciado a la metanarrativa socialista-feminista.

Uno de los motivos más recurrentes en Churchill se encapsula en la palabra "aterrador", pronunciada por muchos de sus personajes, especialmente niños (o, para ser exactos, niñas). Angie en Top Girls (1982) pone fin a la obra cuando despierta en medio de la noche profiriendo este adjetivo, que se ha entendido como símbolo del desamparo en que las políticas neoliberales de Margaret Thatcher en la década de 1980 dejarían a aquellos no equipados para ser "top". Entre Angie y Joan, las niñas que no pueden dormir en los ochenta y el dos mil respectivamente, se sitúan Josie y Lily, madres adolescentes en la obra The Skriker (1994). Josie, que ha matado a su bebé, y Lily, que involuntariamente lo abandonará, son perseguidas por el ente del título, que cambia de forma y discurso dependiendo de la ocasión. The Skriker es quizás la más postmoderna de las obras de Churchill. Candice Amich sugiere que el personaje principal "comanda el espacio y el tiempo de una manera que recuerda a la fluidez del capital multinacional" (394). El análisis de Amich, como antes los de Thomas y Jernigan, pone atención en la supuesta ambigüedad política de Churchill, representada aquí a través de una figura que es "seductora y repelente al mismo tiempo" (398). Sin embargo, su conclusión es que esta obra emplea estructuras postmodernistas para criticar la así llamada condición postmoderna: "Churchill pone en primer plano el terror asociado a la pérdida de la historicidad que acompaña a la compresión espacio-temporal, tratando de sacar a su público del estado letárgico en que se encuentra" (396). Al igual que en Lejos, este intento de recuperar la historicidad se genera a través de una distopía, el 'aterrador' futuro que Lily experimenta. Skriker abusa de la ingenuidad de Lily, llevándola al mundo subterráneo (sin su bebé) por un siglo entero. Cuando regresa conoce a su nieta, que ahora es una vieja, y a su tatara-tatara-nieta, que es una niña deforme. El cuerpo de esta última -como todas las criaturas dañadas en Lejos- representa, según Amich, "el envenenamiento acumulado del planeta" (410).

En Lejos, la cadena distópica se desata por una mentira y supone (contra Foucault) la existencia de una verdad que se ha ocultado. La renuncia de Joan a esa verdad es el primer paso en una espiral que la llevará a convertirse en insensible al sufrimiento de los demás (en el segundo acto) y, finalmente, a recurrir ella misma a la violencia (en el tercero). No es la imposibilidad de 
la verdad sino su abdicación lo que la obra denuncia. Sin negar el vínculo específico con Foucault en Softcops o el uso de técnicas posmodernistas en los años 1990, el trabajo de Churchill aún puede interpretarse apropiadamente desde un enfoque socialista-feminista. Las "lagunas y contradicciones" detectadas por Thomas son la marca de una dramaturga que nunca ha vacilado a la hora de cuestionar supuestos ideológicos, pero cuyas obras todavía vislumbran la posibilidad de cambio social.

\section{UTOPÍA Y DISTOPÍA}

En su libro Churchill's Socialism (2009), Sian Adiseshiah conecta la dramaturgia de esta autora con el desarrollo de ideas progresistas a través del pensamiento utópico, que de una u otra forma está siempre presente en el teatro de Churchill. Como ya se ha indicado, éste muchas veces se manifiesta a través de una distopía o utopía negativa, que actúa como advertencia para cambiar de rumbo mientras exista tiempo. Es importante, sin embargo, distinguir entre distopía y anti-utopía. En el campo literario, la primera se refiere a "una sociedad inexistente [...] que el autor espera que el lector contemporáneo vea como mucho peor que la sociedad en la que vive", mientras que la segunda consiste en "una sociedad inexistente [...] que el autor espera que el lector contemporáneo vea como una crítica al pensamiento utópico" (Sargent 9). La anti-utopía condena tanto la utopía como su reverso, la distopía, en la medida en que ambas son "sueños y pesadillas que se refieren a las formas en que grupos de personas organizan sus vidas, y que por lo general aspiran a una sociedad radicalmente diferente a aquella en la que viven quienes están soñando" (3). En un artículo reciente, Raffaella Baccolini afirma que "nuestra época necesita más que nunca de la utopía, pero parece ser capaz de recuperar la utopía en la mayoría de los casos a través de la distopía" (3). Según Baccolini, el género distópico es especialmente preferido por escritoras feministas, para quienes la "tradición patriarcal [de las utopías clásicas] no constituye una gran pérdida" (2).

El uso de la distopía en Lejos es a la vez repetición y partida: repetición porque el augurio de un futuro aterrador para las nuevas generaciones ha sido tema recurrente para Churchill; partida, porque, a pesar de la brusquedad de sus saltos en el tiempo, la estructura de Lejos sigue (inusualmente en esta dramaturgia) una progresión lineal. En palabras del crítico Irving Wardle, la obra "procede lógicamente, casi uniendo una línea de puntos, hacia un final 
en que toda la creación ha sido arrastrada a la guerra" (1575). Esta anomalía en Churchill puede ser entendida como una adopción del género utópico/ distópico, donde la relación entre causa y efecto es de vital importancia.

Una de las lecciones tanto de la distopía clásica como de la distopía crítica [...] es que el mundo es capaz de ir de mal en peor, no solo en un momento puntual, sino más bien en una compleja serie de pasos que surgen del orden social existente y de las decisiones que la gente toma en él. [...] Otra lección es que todo lo malo que pueda descender sobre nosotros ha sido producido por condiciones sistémicas y opciones humanas que precedieron al momento presente - estas condiciones solamente se pueden cambiar recordando ese proceso y movilizándose en su contra (Baccolini y Moylan 241).

En este contexto, hay una brecha ideológica significativa entre la distopía y la anti-utopía. Como Darko Suvin expresa, "el intertexto de la anti-utopía ha sido históricamente anti-socialismo", en tanto que el de la distopía "ha sido y sigue siendo más bien una forma radical de anti-capitalismo" (189). Ronald Creagh subraya la paradoja de que el marxismo, nacido como antídoto contra el socialismo utópico de Fourier y Owen (que Marx consideraba ingenuo y poco científico) se haya convertido en la encarnación de la utopía moderna y, con ello, en blanco predilecto de filósofos neoliberales como Friedrich Hayek. Así, "la nueva forma de globalización capitalista ha sido acompañada por un coro de pensadores anti-utópicos", para quienes "el destino humano depende del libre mercado" (66-67).

Kritzer interpreta Lejos como anti-utopía, viendo en ella "una especie de parábola que acusa a la izquierda por sus fracasos en el siglo XX" ("Political Currents" 64). En este esquema, la primera parte de la obra sería una metáfora del estalinismo, "en virtud del cual se perjudicó a los que supuestamente se ayudaba, mientras que apologistas defendían el sistema con constantes mentiras". El segundo segmento apuntaría "a la perspectiva estrecha del sindicalismo" y el tercero, al "sectarismo que caracteriza a la izquierda contemporánea, junto con la proliferación caótica de conflictos indescifrables en todo el mundo" (65). Por fascinante que resulte, esta lectura no es del todo coherente, ya que pasa por alto ciertas características primordiales de la obra. Sugerir que el primer acto evoca la Unión Soviética equivale a ignorar la principal estrategia de Churchill, que es la insinuación de que el mundo que se muestra en la obra no está en realidad tan 'lejos'. Tal como Sarah Kane hizo en Blasted, la idea es traer a 'casa' horrores que se piensa solo son 
posibles en otros lugares. En el segundo acto, las quejas mezquinas y fuera de contexto de Jane y Todd podrían considerarse una crítica al sindicalismo, aunque sería más exacto indicar que la actitud indolente de los sombrereros revela los peligros del 'arte por el arte', actitud que dista de ser un defecto de la izquierda. Es más, esta sección aparece como una reivindicación de la filosofía materialista, en tanto que es el divorcio entre la estética y la realidad el que produce resultados brutales (Chaudhuri 132). Por último, la multiplicación de conflictos a nivel global es sin duda el motivo del tercer acto, pero en el período post-Guerra Fría esta tampoco se relaciona específicamente con la fragmentación de la izquierda.

La interpretación anti-utópica de Kritzer concluye que Lejos, al igual que Blasted, ilustra "la ruptura del idealismo" (73). En esta última obra, Cate vuelve desde la zona de guerra para cuidar de Ian, demostrando que el afecto en el ámbito privado es la única respuesta en un mundo que ha abandonado los valores humanos en la vida pública. Según Kritzer, el regreso de Joan para estar con Todd es también "un signo de esperanza", que se basa no en ideales abstractos sino en "el deseo y compromiso personal" (75). Una lectura distópica de Lejos desafía esta conclusión. Con una trama que crece como bola de nieve partiendo de una simple mentira hasta llegar a la destrucción mundial, Lejos advierte contra la falta de ideales, no su exceso. Harper engaña a Joan al tiempo que la consuela en sus brazos como madre sustituta (y mientras los hijos de otros son maltratados bajo el mismo techo); Joan y Todd ignoran las terribles consecuencias de su 'arte' porque están demasiado ocupados con sus propios intereses románticos y laborales. Si privilegiar la expresión del amor individual sobre el amor por la humanidad (y la naturaleza) es la causa del futuro 'aterrador' que presenta la obra, mal podría decirse que son los sentimientos privados los que ofrecen una alternativa positiva. Aquí Churchill se enfrenta nuevamente al dilema de los cocodrilos, antes también abordado por su colega David Edgar:

Una de las acusaciones más perspicaces que los conservadores han lanzado contra la izquierda se refiere a la supuesta contradicción entre el amor por toda la humanidad y la preocupación por las personas que uno conoce. [...] A mí me parece claro que ambas formas de afecto son limitadas e insuficientes. La primera cegó al experimento socialista; la segunda cuestiona las pretensiones morales de la cultura de empresa (Cit. en Painter 119). 
En la distopía post-socialista de Churchill, el péndulo ha oscilado tanto hacia el amor familiar que justifica el abandono total de quienes están más allá de un estrecho círculo. Como declara Aston, la obra defiende la idea de que "la responsabilidad social, no solo personal, debe salir a la luz si se trata de evitar una catástrofe de proporciones globales" (Caryl Churchill 116-17). Desde la perspectiva del feminismo, esta postura implica reexaminar la noción de que "lo personal es político" para admitir que no solamente lo personal sino también lo político deben activarse en aras de una mejor sociedad. En este sentido, la dicotomía que plantea Edgar está ligada al llamado de Seyla Benhabib a complementar una "ética del cuidado" con la necesaria "ética de la justicia". Aquí se encuentra la principal diferencia entre Blasted y Lejos. Mientras que la obra de Kane termina con una retirada hacia el "pensamiento maternal", o lo personal sin lo político, el feminismo materialista de Churchill recuerda que preocuparse por los otros, y no solo los míos, es de vital importancia.

\section{BIBLIOGRAFÍA}

Adiseshiah, Sian. Churchill's Socialism: Political Resistance in the Plays of Caryl Churchill. Newcastle: Cambridge Scholars, 2009.

Amich, Candice. "Bringing the Global Home: The Commitment of Caryl Churchill's The

Skriker". Modern Drama 50.3 (2007): 394-413.

Aston, Elaine. Caryl Churchill. Plymouth: Northcote House, 2001.

Feminist Views on the English Stage: Women Playwrights, 1990-2000. Cambridge: Cambridge University Press, 2003.

Baccolini, Raffaella y Tom Moylan. "Conclusion: Critical Dystopia and Possibilities”. Dark Horizons: Science Fiction and the Dystopian Imagination. Londres: Routledge, 2003.

Baccolini, Raffaella. "Dystopia Matters: On the Use of Dystopia and Utopia”. Spaces of Utopia: An Electronic Journal 3 (2006): 1-4. <http://ler.letras.up.pt>

Benhabib, Seyla. "The Debate Over Women and Moral Theory Revisited". Feminists Read Habermas: Gendering the Subject of Discourse. Ed. Johanna Meehan. Londres: Routledge, 1995. 181-203.

"The Generalized and the Concrete Other: The Kohlberg-Gilligan Controversy and Feminist Theory". Feminism as Critique: Essays on the Politics of Gender in Late-Capitalist Societies. Eds. Seyla Benhabib y Drucilla Cornell. Cambridge: Polity, 1987. 77-95.

"Models of Public Space: Hannah Arendt, the Liberal Tradition, and Jürgen Habermas". Habermas and the Public Sphere. Ed. Craig Calhoun. Londres: MIT Press, 1992. 73-98.

Billington, Michael. Reseña crítica de Far Away, de Caryl Churchill. Guardian (1 diciembre 2000) Theatre Record. 18 noviembre - 1 diciembre 2000. 
Canaday, Margot. "Promising Alliances: The Critical Feminist Theory of Nancy Fraser and

Seyla Benhabib". Feminist Review 74 (2003): 50-69.

Chaudhuri, Una. "Different Hats". Theater 33.3 (2003): 132-134.

Churchill, Caryl. Lejos. Trad. Camila Le-Bert y Alicia del Campo. Manuscrito no publicado, 2010. Plays Two: Softcops, Top Girls, Fen, Serious Money. Londres: Methuen, 1990. The Skriker. Londres: Nick Hern, 1994.

Clapp, Susannah. Reseña crítica de Far Away, de Caryl Churchill. Observer (3 diciembre 2000) Theatre Record. 18 noviembre - 1 diciembre 2000.

Creagh, Ronald. "Anarchism is Back: We May Now Re(Dis)Cover Utopia”. Spaces of Utopia: An Electronic Journal 6 (2007): 61-83. <http://ler.letras.up.pt>

Dietz, Mary G. "Citizenship with a Feminist Face: The Problem with Maternal Thinking". Political Theory 13.1 (1985): 19-37.

Fraser, Nancy. Justice Interruptus: Critical Reflections on the "Postsocialist" Condition. Londres: Routledge, 1997.

"Politics, Culture, and the Public Sphere: Toward a Postmodern Conception". Social Postmodernism: Beyond Identity Politics. Eds. Linda J. Nicholson y Steven Seidman. Cambridge: Cambridge University Press, 1995. 287-312.

Gilligan, Carol. In a Different Voice: Psychological Theory and Women's Development. Cambridge, MA: Harvard University Press, 1982.

Jernigan, Daniel. "Traps, Softcops, Blue Heart, and This is a Chair: Tracking Epistemological Upheaval in Caryl Churchill's Shorter Plays". Modern Drama 47.1 (2004): 21-43.

Kane, Sarah. Complete Plays: Blasted, Phaedra's Love, Cleansed, Crave, 4.48 Psychosis, Skin. Londres: Methuen, 2001.

Koenig, Rhoda. Reseña crítica de Far Away, de Caryl Churchill. Independent (2 diciembre 2000) Theatre Record. 18 noviembre - 1 diciembre 2000.

Kritzer, Amelia Howe. "Political Currents in Caryl Churchill's Plays at the Turn of the Millennium". Crucible of Cultures: Anglophone Drama at the Dawn of a New Millennium. Ed. Marc Maufort. Bruselas: Peter Lang, 2002. 57-67.

Political Theatre in Post-Thatcher Britain: New Writing 1995-2005. Basingstoke: Palgrave Macmillan, 2008.

Le-Bert, Camila. "Theatre". <camilale-bert.com>

Painter, Susan. Edgar: The Playwright. Londres: Methuen, 1996.

Pateman, Carole. "Feminist Critiques of the Public/Private Dichotomy". Feminism and Equality. Ed. Anne Phillips. Oxford: Blackwell, 1987. 103-26.

Sargent, Lyman Tower. "The Three Faces of Utopianism Revisited”. Utopian Studies 5.1 (1994): 1-37.

Spencer, Charles. Reseña crítica de Far Away, de Caryl Churchill. Daily Telegraph (5 diciembre 2000) Theatre Record. 18 noviembre - 1 diciembre 2000.

Suvin, Darko. “Theses on Dystopia 2001”. Dark Horizons: Science Fiction and the Dystopian Imagination. Eds. Raffaella Baccolini y Tom Moylan. Londres: Routledge, 2003. 187-201. 
Thomas, Jane. "The Plays of Caryl Churchill: Essays in Refusal". The Death of the Playwright? Modern British Drama and Literary Theory. Ed. Adrian Page. Basingstoke: Palgrave Macmillan, 1992. 160-85.

Wardle, Irving. Reseña crítica de Far Away, de Caryl Churchill. Sunday Telegraph (3 diciembre 2000) Theatre Record. 18 noviembre - 1 diciembre 2000. 